\title{
Über die weibliche Blüte von Juniperus communis.
}

\author{
Von 0. Renner, München.
}

(Mit 6 Abbildungen im Text.)

Die weibliche Blüte von Juniperus communis, überhaupt der Sektion Oxycedrus, ist bekanntlich dadurch merkwürdig, daß die 3 Samenanlagen, die sich an ihrer Spitze finden, mit dem letzten Blattwirtel des Blütensprosses alternieren. Mit dieser Tatsache, die die Morphologie schon viel beschäftigt hat, findet sich z. B. Eichler ${ }^{1}$ ) in der Weise ab, daß er sagt, jedes der 3 Fruchtblätter - denn als solche betrachtet er die Blätter des letzten Wirtels - trägt eine seitliche Samenanlage. Daß er diese trotzdem als axillär auffaßt, ebenso wie die gepaarten Samenanlagen bei Juniperus Sabina, geht aus einer anderen Stelle ${ }^{2}$ ) hervor, wo es heißt: „Quod denique ovulum interdum haud accurate ante squamae medium positum est (Juniperus), nil impedit, quominus pro organo vere axillari habeamus, quum huius quoque rationis analoga satis sunt cognita“. Eine genauere Vorstellung von dem Zustandekommen der auffallenden Stellungsverhältnisse hat Strasburger ${ }^{3}$ ) sich zu bilden versucht. Er nimmt an, daß ,von den zwei wohl ursprünglich vorhandenen Blüten (= Samenanlagen) jeder Schuppe infolge räumlicher Verhältnisse die eine stets abortierte, allmählich gar nicht mehr zur Entwicklung kam und schließlich in einseitiger Entwicklung konstant vererbt wurde". Eine eigentliche Alternanz der Samenanlagen mit den Fruchtblättern ist demnach bei Eichler und Strasburger nicht zugegeben. Dagegen nimmt $\mathrm{Sachs}^{4}$ ) die Verhältnisse, wie sie sind, ohne durch eine spekulative Konstruktion die Übereinstimmung mit gewissen morphologischen „Gesetzen“ zu erzwingen: jede Samenanlage entspricht in ihrer Stellung einem Blatt, ist echt achsenbürtig wie die Ovula der Primulaceen, Kompositen usw. Und mit aller wünschenswerten Ausführlichkeit und Präzision spricht Schumann ${ }^{5}$ ) sich für diese Auffassung aus. Er erwägt dabei die schon von Stras-

1) Eichler, Blütendiagramme I (1875), pag. 67; Coniferae in Engler u. Prantl, Natürl. Pflanzenfam. II, 1. Abt. (1889), pag. 101.

2) Ders., Coniferae in Martii Flora Brasiliensis, Vol. IV, pars I, 185̄ - -63. Excursus morphologicus de formatione florum Gymnospermarum, pag. 446.

3) Strasburger, Die Coniferen und die Gnetaceen (1872), pag. 32.

4) Sachs, Lehrbuch der Botanik, 2. Aufl. (1870), pag. 424, 404.

5) Schumann, Über die weiblichen Blüten der Coniferen. Verhandl. des Botan. Vereins der Provinz Brandenburg 1902, pag. $72 \mathrm{ff}$. 
burger $\left.{ }^{1}\right)$ gestreifte Möglichkeit, daß die Samenanlagen durch Reduktion von Sporophyllen entstanden sein könnten, wodurch ihre Stellung unter das normale Schema gebracht wäre, weist diese Vorstellung aber ab, mit einer kurzen Motivierung, die sich hauptsächlich auf die nahe Verwandtschaft zwischen Oxycedrus und Sabina gründet. Die Blätter des letzten Wirtels der Blüte sind auch für Sachs und für Schumann echte Karpelle - auf die Kontroverse bezüglich der Unterscheidung von Deckschuppe und Fruchtschuppe einzugehen, ist hier nicht der Ort - und gehören als solche zu den Samenanlagen, wenn eine enge $\mathrm{Zu}$ sammengehörigkeit im morphologischen Sinne auch nicht besteht.

Vor kurzem ist nun $\mathrm{Kubart}^{2}$ ) zu einer Auffassung der weiblichen Blüte von Juniperus-Oxycedrus gekommen, die von der bisher geltenden beträchtlich abweicht. Er studierte die Entwicklungsgeschichte der Blüte, um festzustellen, erstens, ob von den drei hypothetischen abortierten Samenanlagen sich eine Spur nachweisen läßt, zweitens, ob in der Ontogenie tatsächlich eine Verschiebung der ursprünglich blattbürtigen Samenanlagen auf die zwischen den Karpellen gelegenen Achsenteile stattfindet. Das Ergebnis war negativ: Die ersten Anlagen der Ovula stehen in unverkennbarer Alternanz mit den sogenannten Fruchtblättern, und Anlegung abortierender Samenanlagen ist nicht zu beobachten. Damit glaubt Kubart den Nachweis erbracht zu haben, da6 die sogenannten Fruchtblätter als steril, die Samenanlagen als reduzierte Sporophylle aufgefaßt werden müssen. Er beruft sich dabei auf die bekannten Fälle des Vorkommens nackter Samenanlagen bei gewissen Coniferen und auf die zuerst von $\mathrm{Goebel}^{3}$ ) beobachtete Erscheinung, daß in der männlichen Blüte von Juniperus communis der oberste Wirtel von Mikrosporophyllen durch einfache gestielte Pollensäcke repräsentiert wird. Die Wucherung, die nach der Bestäubung auf der Oberseite der sogenannten Fruchtblätter auftritt, wird dann von Kubart nicht als Homologon der Wucherung auf den Karpellen der übrigen Cupressineen, sondern als Element sui generis aufgefaßt und als „Arillargebilde“ bezeichnet. Diese Deutung, um von der gewählten Bezeichnung ganz zu schweigen, kann ich übrigens nicht für eine notwendige Konsequenz der Kubartschen Auffassung der Samenanlagen halten. Auch anderswo beteiligen sich sterile Schuppen an der Zapfen-

1) Strasburger, 1. c. pag. 33 .

2) Bruno Kubart, Die weibliche Blüte von Juniperus communis L. Eine ontogenetisch-morphologische Studie. Sitzungsberichte der kaiserl. Akademie der Wissensch. in Wien, mathem.-naturwiss. Klasse, Bd. CXIV, Abt. 1, pag. 499 ff.

3) Goebel, Organographie der Pflanzen, pag. 695. 
bildung und können zwanglos als gehemmte. Fruchtblätter betrachtet werden, die wohl die Fähigkeit zu ausgiebigem Wachstum und im Zusammenhange damit gewisse Eigentümlichkeiten der Gefäßbündelausbreitung besitzen - auch die sterilen Fruchtblätter von Juniperus (Sect. Sabina) virginiana zeigen nach Strasburger (l. c. p. 33) ein doppeltes Gefäßbündelsystem —, dagegen die Möglichkeit Samenanlagen zu produzieren verloren bezw. nie besessen haben.

In der Literatur glaubt Kubart zwei Autoren $\mathrm{zu}$ finden, die seine Ansicht teilen, daß die Samenanlagen von Juniperus communis reduzierte Sporophylle darstellen. Das eine Zitat bei Kubart (pag. 502) ist Mohl1) entnommen: „Betrachten wir das weibliche Blütenkätzchen von Juniperus, Thuja, Cupressus, so werden wir seine Achse unmittelbar mit Carpellarblättern besetzt und dieselben nicht, wie bei Pinus, in den Achseln von Brakteen stehen finden. Man kann entweder annehmen, daß diese Carpellarblätter von Juniperus die metamorphosierten Blätter der Hauptachse des Kätzchens sind, oder man kann annehmen, daß sie, wie bei Pinus, sekundären Achsen angehören, und daß die ihnen zugehörigen Brakteen fehlgeschlagen sind, oder daß die Brakteen ... mit dem Carpellarblatte aufs innigste verwachsen sind." Und Kubart bemerkt dazu, diese Darstellung enthalte „eine kleine Unrichtigkeit"; denn Deckblätter (= Fruchtblätter) seien bei Juniperus Sektion Sabina, Thuja und Cupressus vorhanden und fehlen nur bei Juniperus Sektion Oxycedrus. Aber Mohl berührt den Gegenstand gar nicht, der Kubart interessiert, und sagt, wie zu erwarten, auch nichts Unrichtiges; die Frage, die er erörtert. ist die nach der Möglichkeit einer Unterscheidung von Deckschuppe und Fruchtschuppe bei den Cupressineen. Nicht besser steht es mit dem zweiten Gewährsmann Kubarts. Sachs ${ }^{2}$ ) schreibt: „Die Samenanlagen alternieren anscheinend mit dem oberen dreigliedrigen Blattquirl und würden so ihrer Stellung nach selbst als metamorphosierte Blätter zù betrachten sein; die Blätter des oberen, mit ihnen alternierenden Quirls schwellen nach der Befruchtung an und bilden die Pulpa der blauen Wacholderbeere ..., sie können daher als Karpelle bezeichnet werden". Wie aus dem weiteren Zusammenhang hervorgeht ${ }^{3}$ ), handelt es sich für Sachs hier nur darum, die morphologische Dignität der Samenknospen festzustellen, zu ent-

1) Mohl, Vermischte Schriften botanischen Inhalts, 1845. IV. Über die männlichen Blüten der Coniferen, pag. 59.

2) Sachs, Lehrbuch der Botanik, 2. Aufl. (18-0), pag. 424.

3) Vergl. ebenda pag. 404: „Häufiger ist es, daß die Samenknospe... in der Stellung einem Blatt entspricht, wie bei Juniperus, den Primulaceen und Compositen". Flora 1907. 
scheiden, ob sie einem Sproß, einem ganzen Blatt oder einem Blatteil gleichwertig sind. Er denkt gar nicht daran, die Samenanlagen von Juniperus communis für reduzierte Sporophylle $\mathrm{zu}$ halten, und die fleischig werdenden Blätter sind für ihn echte Karpelle. Der Begriff Karpell ist eben weder morphologisch noch organographisch streng einheitlich. Denn die Regel, daß die Karpelle Samenanlagen erzeugen, erleidet eine Ausnahme z. B. bei den Primulaceen, die andere, daß sie mit dem Schutz der heranwachsenden Samen betraut sind, bei Cycas. Allerdings müssen wir die Erzeugung der Samenanlagen durch Sporophylle als das Ursprüngliche, die abweichenden Fälle als abgeleitet betrachten.

Kubart steht also, soweit ihm und mir die Literatur bekannt ist, mit seiner Ansicht allein. Und nun fragt es sich, ob sie der älteren gegenüber den Vorzug verdient. In den Ausführungen Schumanns, die Kubart nicht gekannt zu haben scheint, und die auch mir erst nach dem Abschluß meiner Untersuchungen bekannt wurden, ist eigentlich schon eine Widerlegung der Kubartschen Konstruktion enthalten, weil Schumann, wie oben erwähnt, seiner eigenen Deutung eine andere gegenüberstellt, die er ablehnt, und die mit der jetzt von Kubart vertretenen identisch ist. Auf die Begründung der Ablehnung ist im folgenden mehr Sorgfalt verwendet als bei Schumann, ohne daß neue Gesichtspunkte sich ergeben hätten.

Kubart verlangt, daß in der Ontogenie eine Andeutung der Verlagerungs- und Reduktionsvorgänge, ohne die die alte Auffassung nicht denkbar ist, sich müsse ermitteln lassen, wenn diese Auffassung zu Recht besteht. Er scheint aber zu vergessen, daß er für seine Deutung aus der Ontogenie ebensowenig einen Beleg erbringen kann; denn unter den Samenanlagen fehlt jeder Rest der hypothetischen Sporophylle, die sie einst getragen haben sollen, während an den letzten nackten Pollensäcken der männlichen Blüte die tragenden Sporophylle immerhin noch durch einen kurzen Stiel (nebenbei bemerkt ohne Gefäßbündel) repräsentiert sind. Die Entwicklungsgeschichte kann also hier wie in vielen anderen Fällen eine Entscheidung nicht bringen. Von zahllocen Veränderungen, die wir als in der Phylogenie vor sich gegangen postulieren, in erster Linie, wie sehr begreiflich, von Reduktionsvorgängen, ist in der Ontogenie nicht die schwächste Spur erhalten. Wir haben also vorläufig noch gar keinen Anhalt, ob wir die Stellung der Samenanlagen als abgeleitet und ihre Gestalt als ursprünglich betrachten sollen, wie Strasburger und Schumann meinen, oder umgekehrt die Stellung als ursprünglich und die Gestalt als abgeleitet, wie Kubart will. 
Die Samenanlagen sind achsenbürtig und bilden den letzten Wirtel von Ausgliederungen am Blütensproß, daran läßt sich nichts deuteln, wenn man Schumann und Kubart Glauben schenkt. Nach Strasburger wäre immerhin noch ein Zusammenhang zwischen Fruchtblatt und seitlicher Samenanlage zu erkennen, doch kann ich aus der Zeichnung, auf die er verweist, ebensowenig etwas derartiges entnehmen wie aus eigenen Präparaten. Wenn nun ein weiterer akzessorischer Quirl von Ausgliederungen, die wohl rudimentär bleiben müssen, auftritt, so werden die Glieder dieses Wirtels sich in Alternanz mit den Samenanlagen und vor die Fruchtblätter stellen. Einen solchen Fall hat Kubart tatsächlich zu Gesicht bekommen und auf Tafel II in Fig. 10 und 11 abgebildet, und jedenfalls hält er ihn für eine wichtige Stütze seiner Auffassung. Für mich ist dieses Stellungsverhältnis nichts als eine notwendige Konsequenz der Tatsache, daß eben die Samenanlagen am Vegetationspunkt die Stelle von Blättern einnehmen. Die Frage, ob die Samenanlagen aus Sporophyllen durch Reduktion hervorgegangen sind, wird durch die Beobachtung Kubarts, nach meinem Dafürhalten wenigstens, gar nicht berührt.

Der einzige Weg, auf dem unter diesen Umständen eine einigermaßen wahrscheinliche Entscheidung sich erreichen läßt, ist der der vergleichenden Betrachtung des ganzen Verwandtschaftskreises. .Kubart hat, wie es scheint aus prinzipiellen Gründen, auf dieses Hilfsmittel' verzichtet. Und vielleicht legt er aus denselben Gründen der Tatsache kein Gewicht bei, daß das aus der Vergleichung gewonnene Material ohne Ausnahme gegen ihn spricht.

Es wird sich darum handeln, unter den Cupressineen nach Formen zu suchen, bei denen eine Reduktion der weiblichen Sporophylle zu nackten Samenanlagen ebenso unmittelbar zu verfolgen ist wie der entsprechende Vorgang in der männlichen Blüte von Juniperus communis. Am ursprünglichsten dürften die Verhältnisse bei Cupressus ${ }^{1}$ ) und Chamaecyparis sein. Bei der verhältnismäßig großen Zahl von Fruchtblattquirlen sind hier Reduktionsvorgänge am ehesten zu erwarten. Aber wenn der oberste Fruchtblattquirl nicht ganz normal, d. h. fertil ist, erscheint er steril, nie zu nackten Samenanlagen reduziert. Bei Thujopsis, Libocedrus, Thuja ist der oberste Fruchtblattwirtel sogar regelmäßig steril und oft zur sogenannten Columella verwachsen. Bei Fitzroya scheint etwas Ähnliches vorzukommen, bei Actinostrobus erhebt sich zwischen

1) Die Angaben sind Eichler entnommen (1. c. 1889), aber großenteils an Herbarmaterial geprüft. 
den Samen ein ungegliederter Achsenfortsatz, der keine Blattorgane erkennen läßt: Bei Callitris (inkl. Frenela) sind dagegen Verhältnisse zu beobachten, die Kubart allenfalls für seine Auffassung in Anspruch nehmen könnte. Hier findet sich nämlich nach Parlatore ${ }^{1}$ ) (unter Frenela) eine .,columna nunc obsoleta nunc magis minusve elongata, crassa aut angusta, tricruris ant triquetra" und von diesem Gebilde gibt Bentham ${ }^{2}$ ), ebenfalls unter Frenela, an, es sei „,sometimes apparently formed of abortive ovules". Und noch genauer sind die Verhältnisse bei Bentham und Hooker ${ }^{3}$ ) geschildert, wo es von Callitris heißt: „semina ... intima omnino abortiva columnae centrali adnata“. Eine solche „columna“ mit 3 ,abortierten Samen“ aus einem reifen

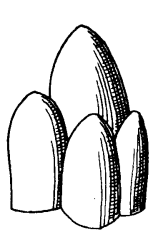

Fig. 1. Columella von Callitris Mülleri, etwa $10 \mathrm{mal}$ vergr. Zapfen von Callitris Mülleri ist ị Fig. 1 dargestellt. Die drei seitlichen Ausgliederungen alternieren genau mit dem letzten Fruchtblattkreis, und wenn sie wirklich Samenanlagen wären, könnte man sie, wollte man $\mathrm{Ku}$ barts Deutung vertreten, vielleicht als zu Samenanlagen reduzierte Sporophylle betrachten. Aber nach einer genaueren Untersuchung der fraglichen Gebilde bei Callitris Mülleri kommt es mir sehr unwahrscheinlich vor, daß sie fehlgeschlagene Samen darstellen. Den Hauptanteil an der Bildung der ellipsoidischen Körper, der 3 seitlichen wie des mittleren terminalen, hat nämlich ein großer Harzraum, der von wenigen Schichten schwach sklerosierten Gewebes und von einer dickwandigen, spaltöffnungsfreien Epidermis überdeckt ist; von Mikropyle und Nucellus ist keine Spur vorhanden. Die „semina abortiva“ sind also nichts anderes als rudimentäre Ausgliederungen am Blütenvegetationspunkt, die man wohl als vergrößerte Fruchtblattprimordien ansehen darf. $\mathrm{Zu}$ dieser Deutung stimmt sehr gut, daß bei Callitris quadrivalvis von den 4 Schuppen, die den Zapfen bilden, die 2 oberen steril sind.

Und genau dieselben Verhältnisse (von der Zahl der Samenanlagen abgesehen) wie bei Callitris quadrivalvis finden wir in der Sektion Sabina von Juniperus, also bei den nächsten Verwandten von JuniperusOxycedrus, auf deren Übereinstimmung es bei der Vergleichung natürlich in erster Linie ankommt. Die Figur 2 stellt eine weibliche Blüte von Juniperus Sabina dar, von oben gesehen. Fertil ist nur der untere der beiden zweigliedrigen Blattwirtel, die den Beerenzapfen bilden,

1) Parlatore, Coniferae in De Candolle Prodromus XVI, pag. 445.

2) Bentham, Flora Australiensis VI (1873), pag. 234.

3) Bentham et Hooker, Genera Plantarum III (1880), pag. 424. 
nicht der obere, wie Kubart (p. 520) angibt. Auch Rikli ${ }^{1}$ ) trifft nicht das Rechte, wenn er meint, ,die (4) Karpelle tragen am Grunde je eine Samenanlage“. Jedes Blatt des unteren Wirtels trägt nämlich in der Achsel ein Paar Samenanlagen (Fig. 2), häufig auch nur eine einzige seitliche, und zwischen den beiden fertilen Karpellen mit ihren Samenanlagen erstreckt sich ein schmaler Geweberücken, der die beiden sterilen, obersten Blätter verbindet und die Spitze der Blütenachse darstellt. Auch bei der dritten Sektion von Juniperus, bei Caryocedrus, wird die weibliche Blüte nach Eichler (Herbarmaterial stand. mir leider nicht zur Verfügung) von drei bis vier dreigliedrigen Schuppenquirlen gebildet, von denen in der Regel nur einer der mittleren fruchtbar ist.

Schumann glaubt für seine Annahme, daß die Samenanlagen von Juniperus communis echt achsenbürtige Organe, aber doch nicht reduzierte Sporophylle seien, eine Beobachtung an Juniperus Sabina ins Feld führen zu müssen, die bei Berg und $\mathrm{Schmidt}^{2}$ ) als einfache Modifikation der Blütenentwicklung mitgeteilt ist, und die auch Schumann nicht als eigentlich teratologisch zu betrachten scheint. Die genannten Autoren wollen nämlich gelegentlich eine einzige mittelständige Samenanlage gefunden haben und bilden einen solchen

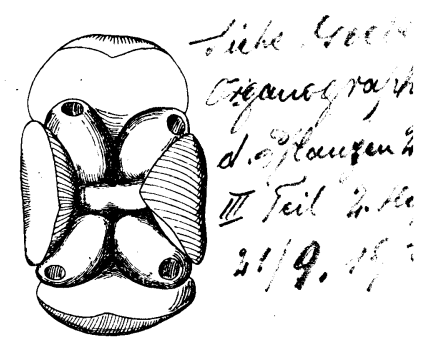

Fig. 2. Weibliche, nicht mehr junge Blüte von Juniperus sabina. Fall im Längsschnitt ab. Daß die Achse zwischen den oberen sterilen Fruchtblättern als keulenförmiger Körper sich fortsetzt, ist nach Schumann nicht sehr selten. Aber wie dieses Achsenende durch eine Samenanlage ersetzt werden soll, wobei die Fruchtblätter sämtlich steril bleiben, ist unverständlich, wenn man die Morphologie der normalen Blüte ins Auge faßt. Wenn derlei Fälle also tatsächlich vorkommen, so müssen sie als Mißbildungen angesehen werden, deren Verwendung für morphologischphylogenetische Spekulationen nicht gerechtfertigt ist. Auch ist die Möglichkeit nicht ausgeschlossen, daß bei Berg ein Beobachtungsfehler vorliegt, weil eine Reduktion der Samenanlagen bis auf eine einzige, natürlich in der Achsel eines unteren Fruchtblattes stehende, hin und wieder zu beobachten sein soll. Jedenfalls kann eine so merkwürdige Mitteilung nicht als sicher gegründet gelten, solange der Autor nicht ausdrücklich auf die Schwierigkeit der morphologischen Deutung hinweist.

1) Rikli, Juniperus sabina in Kirchner, Löw und Schröter, Lebensgeschichte der Blütenpflanzen Mitteleuropas, Bd. I (1906), pag. 332.

2) Berg u. Schmidt, Darstellung sämtlicher offizinellen Gewächse. 1854 bis 1863 . Tafel XXXa. 
Wenn also an der Spitze der weiblichen Blüten der Cupressineen eine Hemmung der Fruchtblattbildung eintritt, so kommt sie immer in einer Sterilisierung zum Ausdruck, nicht in einer Reduktion zu nackten Samenanlagen. Wo sterile Blätter an der Spitze fehlen, wie bei manchen Arten von Cupressus, ist jedenfalls nie ein Übergang zwischen fertilen Fruchtblättern und einzelnen Samenanlagen zu finden, so daf nichts für die Vermutung spricht, die innersten, höchst stehenden Samenanlagen könnten ganzen Sporophyllen gleichwertig sein.

Auch gewisse teratologische Vorkommnisse lassen sich eher für Strasburger verwenden als für Kubart. Daß bei Juniperus Oxycedrus die Einzahl des Fruchtblattwirtels durch Reduktion zustande gekommen sein dürfte, wird durch die Betrachtung der anderen Sektionen von Juniperus und der übrigen Cupressineen von vornherein wahrscheinlich. Tatsächlich ist nicht selten zu beobachten, daß die Zahl der Fruchtblätter an der Beere auf 6 vermehrt ist, was wir wohl als Rückschlag betrachten dürfen. Gewöhnlich sind die Blätter dieses akzessorischen unteren Quirls steril, sie haben also mit den Fruchtblättern nur das eine gemein, daß sie nach der Bestäubung stark wachsen, fleischig werden und so an der Bildung der „Beere“" sich beteiligen; in seltenen Fällen aber werden sie wenigstens zum Teil fertil, d. h. sie tragen in ihren Achseln Samenanlagen. Auf die Einzelheiten dieser Verhältnisse wird unten zurückzukommen sein. Nun findet man in den Blüten der Coniferen nirgends sterile Wirtel zwischen die fertilen eingeschaltet ${ }^{1}$ ), und diese Regel erleidet bei den abnormen Blüten von Juniperus communis keine Ausnahme, wenn man die Blätter des letzten Wirtels als fertile Karpelle betrachtet. Nach Kubarts Auffassung würde dagegen in einer solchen Blüte auf die unteren fertilen Fruchtblätter ein steriler Wirtel folgen und auf diesen wieder ein fertiler, in dem noch dazu die Sporophylle selbst vollständig unterdrückt wären. Es würde eine sprunghafte Änderung der Entwicklungsrichtung von einem Blattkreis zum anderen vorliegen, wie wir sie uns kaum als möglich vorstellen können.

Teratologica können freilich allen Erfahrungssätzen der Morphologie Hohn sprechen. Aber der abnorme Fall fügt sich so ungezwungen in die Reihe der übrigen angezogenen Daten ein, daß ich ihn nicht übergehen möchte, trotzdem ich glaube, da6 die Ansicht Strasburgers auf dieses weitere begründende Moment nicht angewiesen ist.

1) Der von mir in Flora, Bd. 93 (1904), pag. 298 geschilderte Fall, daß in Zwitterblüten von J. communis die Karpelle von den Staubblättern durch eiuen sterilen Blattkreis getrennt sein können, gchört wegen der Ungleichwertigkeit der Sporophylle nicht hierher. 
Viel wichtiger erscheinen mir die Resultate' der vergleichenden Untersuchung. Nach Kubart ist die Beere von Juniperus communis mit der von Jun. Sabina nicht zu vergleichen; Juniperús-Oxycedrus mit seinem "Arillargebilde“" ist ein vollkommen aberranter Typus, ohne Homologie im Kreis der Cupressineen, überhaupt unter den Coniferen. Nach der alten Auffassung gibt es eine einzige Art von Beerenzapfen in den drei Formengruppen, die als Gattung Juniperus zusammengefaßt werden und unbestreitbar mit einander große Ähnlichkeit haben, und die Arten, die sich zunächst an Juniperus communis anschließen, die Arten der Sektion Oxycedrus, sinط eine Formengruppe mit merkwürdig abgeleiteten Stellungsverhältnissen in der weiblichen Blüte, aber eine Gruppe, die durch die Sektionen Sabina und Caryocedrus engen Anschluß an die übrigen Cupressineen gewinnt. Die Konstruktion solcher zusammenhängenden Reihen pflegt dem „systematischen .Gefühl“ mehr Befriedigung zu verschaffen als die Aufstellung isolierter, ohne Brücke weit von einander abstehender Typen, und um etwas weiteres kann es sich bei derlei Fragen ja nicht handeln.

Blüten von Juniperus communis mit einem zweiten fertilen Fruchtblattkreis scheinen noch nicht eingehender beschrieben zu sein. Der, soweit mir bekannt, einzige Autor, der ein solches Vorkommen überhaupt erwähnt, Schröter ${ }^{1}$ ), spricht nur davon, daß der akzessorische Fruchtblattkreis fertil werden kann. Es ist also vielleicht nicht überflüssig,

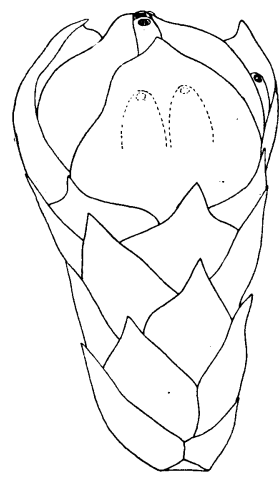

Fig. 3. Weibliche Blüte von J. communis mit 2 fertilen Fruchtblattkreisen. einen derartigen Fall zu schildern. In einer Blüte, die ich vor einigen Jahren fand (Fig. 3-6), trugen zwei Blätter des unteren Wirtels je zwei Samenanlagen in der Achsel, das dritte Blatt war steril, und auf dem Gipfel der Blüte waren wie normal drei Samenanlagen ausgebildet. In einer anderen trug ein Blatt des unteren Kreises zwei Samenanlagen, das zweite eine einzige, das letzte war wieder steril. Die Samenanlagen des akzessorischen Wirtels waren bedeutend kleiner als die gipfelständigen, aber im übrigen ziemlich normal. Fig. 4 stellt einen Längsschnitt dar, der die Blütenspitze annähernd median getroffen hat. In der Lücke zwischen den beiden Fruchtblättern, die mit $c_{1}$ zu-

1) Kirchner, Löw u. Schröter, Lebensgeschichte der Blütenpflanzen Mitteleuropas, Bd. I (1906), pag. 301. - Meine Angabe in Flora, Bd. 93 (1904), pag. 300, daß Parlatore entsprechende Fälle gekannt habe, beruht auf einem Mißverständnis, wie schon Kubart bemerkt hat. 
sammen den oberen Wirtel bilden, stößt eine zu $c_{2}$ gehörende Samenanlage $o_{2}$ unmittelbar an die Samenanlage $o_{1}$ an; ebenso in Fig. 6. In Fig. $\tilde{5}$ ist ein tangentialer Längsschnitt wiedergegeben; von den Paaren von Samenanlagen, die zu den beiden Fruchtblättern $c_{2}$ gehören, ist je eine getroffen, das Fruchtblatt $c_{1}$ ist ziemlich weit außen angeschnitten.

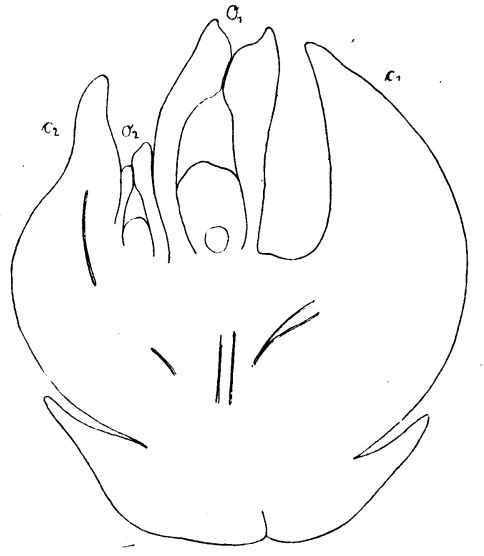

Fig. 4.

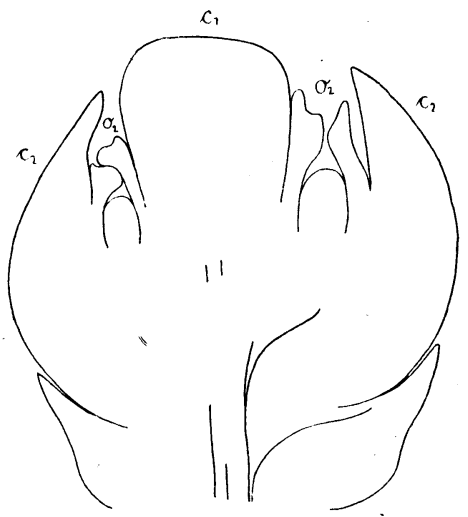

Fig. 5 .

Fig. 4-6. Längsschnitte durch die in Fig. 3 dargestellte Blüte. $c_{1}$ Fruchtblätter des oberen Kreises, $o_{1}$ dazu gehörende Samenanlagen; $c_{2}$ Fruchtblätter des unteren Wirtels, $o_{2}$ die Samenanlagen in deren Achseln.

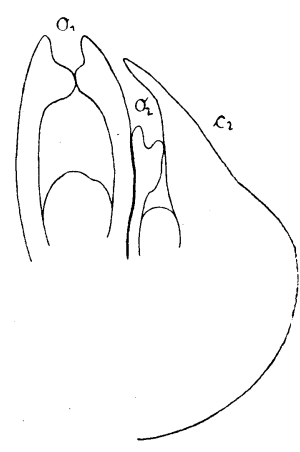

Fig. 6.

Bei der Samenanlage $o_{2}$ in Fig. 6 ist das Integument nur auf der dem Fruchtblatt abgewendeten Seite zur Ausbildung gekommen; der Ringwulst am Innenrand des Integuments, der sonst die Mikropyle verschließt, ist deshalb durch eine einseitige Wucherung ersetzt, die sich an das Fruchtblatt andrängt. $\mathrm{Ob}$ aus den akzessorischen Samenanlagen reife Samen hervorgehen können, wurde noch nicht festgestellt.

Das Auftreten gepaarter axillärer Samenanlagen bei Juniperus communis macht. den Zusammenhang mit der Sektion Sabina besonders eng und kann auch als Stütze für die Annahme Strasburgers herangezogen werden. Es ist aber $\mathrm{zu}$ bedenken, daß bei Biota die Zahl der Samenanlagen im oberen fertilen Quirl auf 1 pro Fruchtblatt reduziert ist. während die Blätter des unteren Kreises je 2 tragen, und so bleibt die Möglichkeit offen, daß der jetzt einzige Fruchtblattwirtel von Juniperus communis nie mehr als drei Samenanlagen produziert hat. Freilich ist der Verschiebungsvorgang leichter vorzustellen, wenn man mit Strasburger von drei Paaren von Samenanlagen ausgeht. 